\title{
A UNIFIED THEORY FOR WEAK SEPARATION PROPERTIES
}

\author{
MAHIDE KÜÇÜK and IDRIS ZORLUTUNA
}

(Received 7 June 1999 and in revised form 3 November 1999)

\begin{abstract}
We devise a framework which leads to the formulation of a unified theory of normality (regularity), semi-normality (semi-regularity), $s$-normality ( $s$-regularity), feeblynormality (feebly-regularity), pre-normality (pre-regularity), and others. Certain aspects of theory are given by unified proof.
\end{abstract}

Keywords and phrases. Unification, normality, regularity, $T_{0}$-identification space.

2000 Mathematics Subject Classification. Primary 54C10, 54D30, 54D20, 54D25.

1. Introduction. Normality and regularity are some of the important properties for studying topological spaces. Several types of normality and regularity occur in the literature. In this paper, we apply the concept of $\varphi$-operation which was defined by Csaszar [7] to unify and generalize several characterizations and properties of a lot of already existing weaker forms of normality and regularity. In Section 2, we introduce the concept of $\varphi \psi$-normality and $\varphi \psi$-regularity as a generalization of the concepts of normality, regularity, semi-normality [17], semi-regularity [14], $s$-normality [23], $s$-regularity [21], feebly-normality [18], feebly-regularity [18], $\beta$-normality [25], $\beta$-regularity [2], and $p$-regularity [19]. Also, we introduce the concepts of $\varphi-R_{0}$ and $\varphi$ - $T_{0}$-identification spaces as generalizations of the concepts of $R_{0}$-space [10], $T_{0^{-}}$ identification space [28], semi- $R_{0}$-space [22], semi- $T_{0}$-identification space [14], and $s$-feebly- $R_{0}$-space [16] to unify and generalize several characterizations. In Section 3, characterizations and properties of $\varphi \psi$-normality and $\varphi \psi$-regularity are investigated.

Throughout the paper, $(X, \tau)$ and $(Y, \vartheta)$ mean topological spaces on which no separation axioms are assumed unless otherwise explicitly stated. Let $A$ be a subset of $X, A$ is called semi-open [20] (respectively, pre-open [26], feebly open [24], and $\beta$-open [1]) if $A \subseteq \operatorname{cl}(\operatorname{int} A)$ (respectively, $A \subseteq \operatorname{int}(\operatorname{cl} A), A \subseteq \operatorname{scl}(\operatorname{int} A)$, and $A \subseteq \operatorname{cl}\left(\operatorname{int}(\operatorname{cl} A)\right.$ ). Semi- $T_{i}$ [22] (respectively, pre- $T_{i}$, feebly- $T_{i}$ [18], and $\beta$ - $T_{i}$ [25]) spaces are defined as ordinary ones except each open set is replaced by semi-open (respectively, pre-open, feebly open, and $\beta$-open) one, $i=0,1$. A space $(X, \tau)$ is $R_{0}$ [10] if and only if for each $O \in \tau$ and $x \in O, \operatorname{cl}\{x\} \subseteq O .(X, \tau)$ is semicompact [12] if for every semi-open cover of it has a finite subcover. A space $(X, \tau)$ is extremally disconnected (E.D.) [5, 6] if $\operatorname{cl} O \in \tau$ for each $O \in \tau$ and is submaximal $[5,6]$ if all dense sets in it are open. A space $(X, \tau)$ is $s$ weakly Hausdorff [11] if for each pair $x, y \in X$ such that $\operatorname{cl}\{x\} \neq \operatorname{cl}\{y\}$, there exist disjoint semi-open sets $U$ and $V$ such that $x \in U$ and $y \in V$. A space $(X, \tau)$ is $R_{1}$ [8] (semi$R_{1}$ [9]) if and only if for each pair $x, y \in X \operatorname{such}$ that $\operatorname{cl}\{x\} \neq \operatorname{cl}\{y\}(\operatorname{scl}\{x\} \neq \operatorname{scl}\{y\})$, there exist disjoint open (semi-open) sets $U$ and $V$ such that $\operatorname{cl}\{x\} \subseteq U$ and $\operatorname{cl}\{y\} \subseteq V$ $(\operatorname{scl}\{x\} \subseteq U$ and $\operatorname{scl}\{y\} \subseteq V)$. 


\section{A unified framework}

DEFINITION 2.1 (see [7]). Let $(X, \tau)$ be a topological space. A mapping $\varphi: P(X) \rightarrow$ $P(X)$ is called an operation on $P(X)$, where $P(X)$ denotes the family all of the subsets of $X$ if and only if for each $A \in P(X)-\{\varnothing\}$, int $A \subseteq A^{\varphi}$ and $\varnothing=\varnothing^{\varphi}$, where $A^{\varphi}$ is denotes the value of $\varphi$ in $A$. The class of all operations on $P(X)$ is denoted by $O(X)$.

Throughout the paper, all of the operations on $P(X)$ are assumed to be monotonous (i.e., such that $A \subseteq B$ implies $A^{\varphi} \subseteq B^{\varphi}$ ).

DefinITION 2.2 (see [7]). Let $(X, \tau)$ be a topological space, $G, H \in P(X)$, and $\varphi \in$ $O(X)$. Then

(i) $G$ is called $\varphi$-open if and only if $G \subseteq G^{\varphi}$.

(ii) The subset $H$ is called $\varphi$-closed if and only if $X-H$ is $\varphi$-open.

The class of all $\varphi$-open ( $\varphi$-closed) subsets of $X$ is denoted by $\varphi O(X)(\varphi C(X))$. For each $x \in X$, the set $\{V \subseteq X \mid x \in V \in \varphi O(X)\}$ is denoted by $N(x, \varphi O(X))$.

DeFinItion 2.3 (see [7]). Let $(X, \tau)$ be a topological space, $G \in P(X)$, and $\varphi \in$ $O(X)$.

(i) The intersection of all $\varphi$-closed sets containing $G$ is the $\varphi$-closure of $G$, denoted by $\varphi \operatorname{cl} G$.

(ii) The union of all $\varphi$-open subsets of $G$ is the $\varphi$-interior of $G$, denoted by $\varphi \operatorname{int} G$.

The set $\varphi \operatorname{cl} G$ is the smallest $\varphi$-closed set containing $G$, and the set $\varphi \operatorname{int} G$ is the largest $\varphi$-open subset of $G$.

In a topological space, if $\varphi=\mathrm{int}$, then $\varphi \mathrm{cl}=\mathrm{cl}$. Similarly, if $\varphi=\mathrm{int} \circ \mathrm{cl}$, then $\varphi \mathrm{cl}=$ $\mathrm{pcl}$, where pcl denotes pre-closure.

The following lemma is obvious above definitions.

LEMMA 2.4. Let $(X, \tau)$ be a topological space, $G \in P(X), \varphi \in O(X)$, and $x \in X$.

(i) $x \in \varphi \operatorname{cl} G \Leftrightarrow \forall T \in N(x, \varphi O(X))(G \cap T \neq \varnothing)$.

(ii) $x \in \varphi \operatorname{int} G \Leftrightarrow \exists T \in N(x, \varphi O(X))(x \in T \subseteq G)$.

DEFINITION 2.5. A topological space $(X, \tau)$ is

(i) a $\varphi-T_{0}$-space if, for any two distinct points $x$ and $y$ of $X$, there exists either a $\varphi$-open set containing $x$ but not $y$ or a $\varphi$-open set containing $y$ but not $x$.

(ii) a $\varphi-T_{1}$-space if, for any two distinct points $x$ and $y$ of $X$, there exists a $\varphi$-open set containing $x$ but not $y$ and a $\varphi$-open set containing $y$ but not $x$.

DEFINITION 2.6. Let $(X, \tau)$ be a topological space, and $\varphi, \psi \in O(X)$.

(i) $(X, \tau)$ is called $\varphi \psi$-normal if and only if for each pair of disjoint $\varphi$-closed sets $A$ and $B$, there exist disjoint $\psi$-open sets $U$ and $V$ such that $A \subseteq U$ and $B \subseteq V$.

(ii) $(X, \tau)$ is called $\psi$-normal if and only if $\varphi=\psi$.

DEFINITION 2.7. Let $(X, \tau)$ be a topological space, and $\varphi, \psi \in O(X)$.

(i) $(X, \tau)$ is called $\varphi \psi$-regular if and only if for each $\varphi$-closed set $A$ and $x \notin A$, there exist disjoint $\psi$-open sets $U$ and $V$ such that $x \in U$ and $A \subseteq V$.

(ii) $(X, \tau)$ is called $\psi$-regular if and only if $\varphi=\psi$.

DEFINITION 2.8. Let $(X, \tau)$ be a topological space and $\varphi \in O(X) .(X, \tau)$ is called $\varphi-R_{0}$ if and only if for each $O \in \varphi O(X)$ and $x \in O, \varphi \operatorname{cl}\{x\} \subseteq O$. 
TABLE 2.1.

\begin{tabular}{|c|c|c|c|c|c|c|}
\hline$\varphi$-ope. & $\psi$-оре. & $\begin{array}{l}\varphi \psi \text {-normal } \\
\text { space }\end{array}$ & $\begin{array}{l}\varphi \psi \text {-regular } \\
\text { space }\end{array}$ & $\begin{array}{l}\varphi-R_{0} \\
\text { space }\end{array}$ & $\begin{array}{l}\varphi-T_{0} \\
\text { identification } \\
\text { space }\end{array}$ & $\begin{array}{l}\psi-T_{0} \\
\text { identification } \\
\text { space }\end{array}$ \\
\hline int & int & normal & regular & $R_{0}[10]$ & $T_{0}$-iden. [28] & $T_{0}$-iden. [28] \\
\hline $\mathrm{cl} \circ$ int & $\mathrm{cl} \circ$ int & $\begin{array}{l}\text { semi- } \\
\text { normal } \\
{[17]}\end{array}$ & $\begin{array}{l}\text { semi- } \\
\text { regular } \\
{[14]}\end{array}$ & $\begin{array}{l}\text { semi- } R_{0} \\
{[22]}\end{array}$ & $\begin{array}{l}\text { semi- } T_{0} \text {-iden. } \\
{[13]}\end{array}$ & $\begin{array}{l}\text { semi- } T_{0} \text {-iden. } \\
{[13]}\end{array}$ \\
\hline int $\circ \mathrm{cl}$ & int $\circ \mathrm{cl}$ & $\begin{array}{l}\text { pre-normal } \\
{[27]}\end{array}$ & pre-regular & $s$-pre- $R_{0}$ & pre- $T_{0}$-iden. & pre- $T_{0}$-iden. \\
\hline $\mathrm{scl} \circ$ int & $\mathrm{scl} \circ$ int & $\begin{array}{l}s \text {-feebly- } \\
\text { normal }\end{array}$ & $\begin{array}{l}s \text {-feebly- } \\
\text { regular }\end{array}$ & $\begin{array}{l}s \text {-feebly- } R_{0} \\
{[16]}\end{array}$ & $\begin{array}{l}\text { feebly- } T_{0}{ }^{-} \\
\text {iden. }\end{array}$ & $\begin{array}{l}\text { feebly- } T_{0}- \\
\text { iden. }\end{array}$ \\
\hline $\mathrm{cl} \circ$ int $\circ \mathrm{cl}$ & $\mathrm{cl} \circ \mathrm{int} \circ \mathrm{cl}$ & $s$ - $\beta$-normal & $s$ - $\beta$-regular & $s-\beta-R_{0}$ & $\beta-T_{0}$-iden. & $\beta-T_{0}$-iden. \\
\hline int & cl॰int & $\begin{array}{l}s \text {-normal } \\
{[23]}\end{array}$ & $\begin{array}{l}s \text {-regular } \\
{[21]}\end{array}$ & $R_{0}[10]$ & $T_{0}$-iden. [28] & $\begin{array}{l}\text { semi- } T_{0} \text {-iden. } \\
{[13]}\end{array}$ \\
\hline int & int $\circ \mathrm{cl}$ & $p$-normal & $\begin{array}{l}p \text {-regular } \\
{[19]}\end{array}$ & $R_{0}[10]$ & $T_{0}$-iden. [28] & pre- $T_{0}$-iden. \\
\hline int & scl $\circ$ int & $\begin{array}{l}\text { feebly- } \\
\text { normal } \\
{[18]}\end{array}$ & $\begin{array}{l}\text { feebly- } \\
\text { regular } \\
{[18]}\end{array}$ & $R_{0}[10]$ & $T_{0}$-iden. [28] & $\begin{array}{l}\text { feebly- } T_{0}- \\
\text { iden. }\end{array}$ \\
\hline int & $\mathrm{cl} \circ$ int $\circ \mathrm{cl}$ & $\begin{array}{l}\beta \text {-normal } \\
{[25]}\end{array}$ & $\begin{array}{l}\beta \text {-regular } \\
{[2]}\end{array}$ & $R_{0}[10]$ & $T_{0}$-iden. [28] & $\beta-T_{0}$-iden. \\
\hline
\end{tabular}

DEFINITION 2.9. Let $(X, \tau)$ be a topological space and $\varphi \in O(X)$. Let $R$ be the equivalence relation on the space $(X, \tau)$ defined by $x R y$ if and only if $\varphi \operatorname{cl}\{x\}=$ $\varphi \operatorname{cl}\{y\}$. The $\varphi-T_{0}$-identification space of $(X, \tau)$ is $\left(X_{\varphi}, Q\left(X_{\varphi}\right)\right)$, where $X_{\varphi}$ is the set of equivalence classes of $R$ and $Q\left(X_{\varphi}\right)$ is the decomposition topology on $X_{\varphi}$. Let $P_{\varphi}:(X, \tau) \rightarrow\left(X_{\varphi}, Q\left(X_{\varphi}\right)\right)$ denote the natural map.

Table 2.1 lists the type of $\varphi \psi$-normal, $\varphi \psi$-regular, $\varphi-R_{0}$, and $\varphi(\psi)$ - $T_{0}$-identification spaces induced by operations $\varphi$ and $\psi$, and gives explicitly the weak forms of normality and regularity to which they correspond. Besides, some new definitions appear in the developing of the unified theory.

\section{Characterizations and properties of $\varphi \psi$-normality and $\varphi \psi$-regularity}

THEOREM 3.1. A space $(X, \tau)$ is $\varphi \psi$-normal if and only if for each $\varphi$-closed set $A$ and $\varphi$-open set $U$ containing $A$, there exists a $\psi$-open set $G$ such that $A \subseteq G \subseteq \psi \operatorname{cl} G \subseteq U$.

Proof. $(\Rightarrow)$ Let $A$ be a $\varphi$-closed set and $U$ be a $\varphi$-open set containing $A$. Then $A \cap(X-U)=\varnothing$ and $X-U$ is a $\varphi$-closed set. There exist $\psi$-open sets $G$ and $H$ such that $A \subseteq G$ and $X-U \subseteq H$. Therefore, we have $A \subseteq G \subseteq X-H \subseteq U$ and hence $\psi \operatorname{cl} G \subseteq$ $\psi \operatorname{cl}(X-H) \subseteq U$ since $X-H$ is $\psi$-closed. Consequently, we obtain $A \subseteq G \subseteq \psi \operatorname{cl} G \subseteq U$.

$(\Leftarrow)$ Let $A$ and $B$ be disjoint $\varphi$-closed sets. Then $A \subseteq X-B$ and $X-B$ is a $\varphi$-open set. There exists a $\psi$-open set $G$ such that $A \subseteq G \subseteq \psi \mathrm{cl} G \subseteq X-B$. Therefore, we have $A \subseteq G, B \subseteq X-\psi \operatorname{cl} G$ and $G \cap(X-\psi \operatorname{cl} G)=\varnothing$. This shows that $X$ is $\varphi \psi$-normal. 
THEOREM 3.2. The following properties are equivalent for a space $X$ :

(1) $X$ is $\varphi \psi$-regular;

(2) for each $x \in X$ and $U \in \varphi O(X)$ such that $x \in U$, there exists a $\psi$-open set $V$ such that $x \in V \subseteq \psi \mathrm{cl} V \subseteq U$;

(3) for each $\varphi$-closed set $F$ of $X, F=\cap\{\psi \mathrm{cl} W \mid F \subseteq W, W \in \psi O(X)\}$;

(4) for each subset $A \subseteq X$ and each $\varphi$-open set $U$ such that $A \cap U \neq \varnothing$, there exists $W \in \psi O(X)$ such that $A \cap W \neq \varnothing$ and $\psi \mathrm{cl} W \subseteq U$;

(5) for each nonempty subset $A \subseteq X$ and each $\varphi$-closed set $F$ of $X$ such that $A \cap F=$ $\varnothing$, there exist $U, W \in \psi O(X)$ such that $A \cap U \neq \varnothing, F \subseteq W$, and $U \cap W=\varnothing$.

Proof. $(1) \Rightarrow(2)$. Let $U$ be a $\varphi$-open set containing $x$, then $X-U$ is $\varphi$-closed set in $X$ and $x \notin X-U$. By (1), there are two disjoint $\psi$-open sets $V, H$ such that $x \in V$ and $X-U \subseteq H$. Therefore, we have $x \in V \subseteq X-H \subseteq U$ and hence $x \in V \subseteq \psi \mathrm{cl} V \subseteq U$.

(2) $\Rightarrow(3)$. Let $F$ be a $\varphi$-closed set of $X$. It is obvious that $F \subseteq \cap\{\psi \operatorname{cl} W \mid F \subseteq W, W \in$ $\psi O(X)\}$. Conversely, let $x \notin F$ and $F \in \varphi C(X)$. Then $X-F$ is $\varphi$-open set containing $x$, by (2) there is a $\psi$-open set $V$ such that $x \in V \subseteq \psi \operatorname{cl} V \subseteq X-F$. Put $W=X-\psi \operatorname{cl} V$, it follows that $F \subseteq W \in \psi O(X)$ and $x \notin \psi \operatorname{cl} W$. This implies that $\cap\{\psi \operatorname{cl} W \mid F \subseteq W, W \in$ $\psi O(X)\} \subseteq F$.

(3) $\Rightarrow(4)$. Let $U$ be $\varphi$-open set in $X$ and $A \cap U \neq \varnothing$. For $x \in A \cap U, X-U$ is $\varphi$-closed set not containing $x$, by (3) there is a $V \in \psi O(X)$ such that $X-U \subseteq V$ and $x \notin \psi \operatorname{cl} V$. Put $W=X-\psi \operatorname{cl} V$, we obtain $W \in \psi O(X), x \in W \cap A \neq \varnothing$ and $\psi \operatorname{cl} W \subseteq \psi \operatorname{cl}(X-V)=$ $X-V \subseteq U$.

$(4) \Rightarrow(5)$. Let $F$ be $\varphi$-closed set in $X$ and $A \cap F=\varnothing$, where $A \neq \varnothing$. Since $X-F$ is $\varphi$ open in $X$ and $A$ is nonempty, by (4) there is a $W \in \psi O(X)$ such that $A \cap W \neq \varnothing$ and $\psi \mathrm{cl} W \subseteq X-F$. Put $V=X-\psi \operatorname{cl} W$. Then $F \subseteq V \in \psi O(X)$ and $W \cap V=\varnothing$.

(5) $\Rightarrow(1)$. It is obvious.

THEOREM 3.3. If $(X, \tau)$ is $\varphi \psi$-normal and $\varphi-R_{0}$, then $(X, \tau)$ is $\varphi \psi$-regular.

Proof. Let $A$ be a $\varphi$-closed set not containing $x$. Then $x \in X-A$ is $\varphi$-open set, which implies $\varphi \operatorname{cl}\{x\} \subseteq X-A$, and there exist disjoint $\psi$-open sets $U$ and $V$ such that $x \in \varphi \operatorname{cl}\{x\} \subseteq U$ and $A \subseteq V$.

Referring to Table 2.1, Theorem 3.3 contains several results in the literature. For example, with $\varphi=\psi=\mathrm{cl} \circ$ int it gives that if $(X, \tau)$ is semi-normal, semi- $R_{0}$, then $(X, \tau)$ is semi-regular, a result due to Dorsett [17]. Similarly, with $\varphi=$ int, $\psi=\operatorname{cl} \circ$ int it yields a result due to Maheshwari and Prasad [23].

Example 3.12 [14] shows that the converse of Theorem 3.3 is false.

THEOREM 3.4. The natural map $P_{\varphi}:(X, \tau) \rightarrow\left(X_{\varphi}, Q\left(X_{\varphi}\right)\right)$ is closed, open, and $P_{\varphi}^{-1}\left(P_{\varphi}(O)\right)=O$ for all $O \in \varphi O(X)$ and $\left(X_{\varphi}, Q\left(X_{\varphi}\right)\right)$ is $\varphi-T_{0}$.

Proof. For each $x \in X$, let $C_{x}$ be the equivalence class of $R$ containing $x$. Let $O \in \varphi O(X)$ and $x \in P_{\varphi}^{-1}\left(P_{\varphi}(O)\right)$. Then there exists $y \in O$ such that $C_{x}=C_{y}$. Thus $\varphi \operatorname{cl}\{x\}=\varphi \operatorname{cl}\{y\}$ and $O \in \varphi O(X)$ such that $y \in O$, which implies $x \in O$. Hence $P_{\varphi}^{-1}\left(P_{\varphi}(O)\right) \subseteq O$, which implies $O=P_{\varphi}^{-1}\left(P_{\varphi}(O)\right)$. Since $\tau \subseteq \varphi O(X)$, then $P_{\varphi}^{-1}\left(P_{\varphi}(U)\right)=$ $U$ for all $U \in \tau$, which implies $P_{\varphi}$ is closed and open.

Let $A, B \in X_{\varphi}$ such that $A \neq B$ and let $x \in A$ and $y \in B$. Then $\varphi \operatorname{cl}\{x\} \neq \varphi \operatorname{cl}\{y\}$, 
which implies $x \notin \varphi \operatorname{cl}\{y\}$ or $y \notin \varphi \operatorname{cl}\{x\}$, say, $x \notin \varphi \operatorname{cl}\{y\}$. Since $P_{\varphi}$ is continuous and open, then $A \in D=P_{\varphi}(X-\varphi \operatorname{cl}\{y\}) \in \varphi O\left(X_{\varphi}, Q\left(X_{\varphi}\right)\right)$ and $B \notin D$.

Using Table 2.1, Theorem 3.4 unifies several known results. For example, if $\varphi=$ int, then it yields Theorem 2.1 of Dorsett [10]. Similarly, with $\varphi=\mathrm{cl} \circ$ int it yields Theorem 2.1 of Dorsett [13].

THEOREM 3.5. The following are equivalent:

(a) $(X, \tau)$ is $\varphi-R_{0}$,

(b) $X_{\varphi}=\{\varphi \operatorname{cl}\{x\} \mid x \in X\}$,

(c) $\left(X_{\varphi}, Q\left(X_{\varphi}\right)\right)$ is $\varphi-T_{1}$.

Proof. (a) $\Rightarrow$ (b). Let $C \in X_{\varphi}$ and $x \in C$. If $y \in C$, then $y \in \varphi \operatorname{cl}\{y\}=\varphi \operatorname{cl}\{x\}$, which implies $C \subseteq \varphi \operatorname{cl}\{x\}$. If $y \in \varphi \operatorname{cl}\{x\}$, then $x \in \varphi \operatorname{cl}\{y\}$. Since otherwise, $x \in$ $X-\varphi \operatorname{cl}\{y\} \in \varphi O(X)$, which implies $\varphi \operatorname{cl}\{x\} \subseteq X-\varphi \operatorname{cl}\{y\}$, which is a contradiction. Thus, if $y \in \varphi \operatorname{cl}\{x\}$, then $x \in \varphi \operatorname{cl}\{y\}$, which implies $\varphi \operatorname{cl}\{y\}=\varphi \operatorname{cl}\{x\}$ and $y \in C$.

(b) $\Rightarrow$ (c). Let $A, B \in X_{\varphi}$ such that $A \neq B$. Then there exist $x, y \in X$ such that $A=$ $\varphi \operatorname{cl}\{x\}$ and $B=\varphi \operatorname{cl}\{y\}$, and $\varphi \operatorname{cl}\{x\} \cap \varphi \operatorname{cl}\{y\}=\varnothing$. Then $A \in C=P_{\varphi}(X-\varphi \operatorname{cl}\{y\}) \in$ $\varphi O\left(X_{\varphi}, Q\left(X_{\varphi}\right)\right)$ and $B \notin C$.

(c) $\Rightarrow(\mathrm{a})$. Let $O \in \varphi O(X, \tau)$ and let $x \in O$. Let $y \notin O$ and let $C_{x}, C_{y} \in X_{\varphi}$ containing $x, y$, respectively. Then $x \notin \varphi \operatorname{cl}\{y\}$, which implies $C_{x} \neq C_{y}$ and there exists a $\varphi$ open set $A$ such that $C_{y} \in A$ and $C_{x} \notin A$. Since $P_{\varphi}$ is continuous and open, then $y \in B=P_{\varphi}^{-1}(A) \in \varphi O(X, \tau)$ and $x \notin B$, which implies $y \notin \varphi \operatorname{cl}\{x\}$. Thus $\varphi \operatorname{cl}\{x\} \subseteq O$.

According to Table 2.1, Theorem 3.5 represents the unification of various results in the literature. For example, if $\varphi=\mathrm{cl} \circ$ int, we get a result pertaining to semi- $R_{0}$ space. Thus, the following are equivalent: (a) $(X, \tau)$ is semi- $R_{0}$, (b) $X_{s}=\{\operatorname{scl}\{x\} \mid x \in X\}$, (c) $\left(X_{s}, Q\left(X_{s}\right)\right)$ is semi- $T_{1}\left[13\right.$, Theorem 2.2]. The classical result pertaining to $R_{0}$ space follows by substituting int for $\varphi$.

THEOREM 3.6. A space $(X, \tau)$ is $\varphi \psi$-normal if and only if its $\psi-T_{0}$-identification space $\left(X_{\psi}, Q\left(X_{\psi}\right)\right)$ is $\varphi \psi$-normal, where $\varphi \leq \psi$.

Proof. $(\Rightarrow)$ Let $A$ and $B$ be disjoint $\varphi$-closed sets in $X_{\psi}$. Since $P_{\psi}$ is continuous, open and $\varphi \leq \psi, P_{\psi}^{-1}(A)$ and $P_{\psi}^{-1}(B)$ are disjoint $\varphi$-closed sets in $X$. Then there exist disjoint $\psi$-open sets $U$ and $V$ such that $P_{\psi}^{-1}(A) \subseteq U$ and $P_{\psi}^{-1}(B) \subseteq V$. Since $P_{\psi}$ is continuous, open and $P_{\psi}^{-1}\left(P_{\psi}(U)\right)=U$ for all $U \in \psi O(X)$, then $P_{\psi}(U)$ and $P_{\psi}(V)$ are disjoint $\psi$-open sets in $X_{\psi}$ such that $A \subseteq P_{\psi}(U)$ and $B \subseteq P_{\psi}(V)$.

$(\Leftrightarrow)$ Let $A$ and $B$ be disjoint $\varphi$-closed sets in $X$. Since $P_{\psi}$ is continuous, open and $P_{\psi}^{-1}\left(P_{\psi}(C)\right)=C$ for all $C \in \psi O(X), P_{\psi}(A)$ and $P_{\psi}(B)$ are disjoint $\varphi$-closed sets in $X_{\psi}$. Then there exist disjoint $\psi$-open sets $U$ and $V$ in $X_{\psi}$ such that $P_{\psi}(A) \subseteq U$ and $P_{\psi}(B) \subseteq V$. Since $P_{\psi}$ is continuous and open, then $P_{\psi}^{-1}(U)$ and $P_{\psi}^{-1}(V)$ are disjoint $\psi$-open sets in $X$ containing $A$ and $B$, respectively.

Referring to Table 2.1, Theorem 3.6 contains several results in the literature. For example, with $\varphi=\psi=\mathrm{cl} \circ$ int it gives that a space $(X, \tau)$ is semi-normal if and only if its semi- $T_{0}$-identification space $\left(X_{s}, Q\left(X_{s}\right)\right)$ is semi-normal, a result due to Dorsett [17]. Similarly, with $\varphi=$ int, $\psi=\mathrm{cl} \circ$ int it gives that a space $(X, \tau)$ is $s$-normal if and only if its semi- $T_{0}$-identification space $\left(X_{s}, Q\left(X_{s}\right)\right)$ is $s$-normal, a result due to Dorsett [15]. 
THEOREM 3.7. A space $(X, \tau)$ is $\varphi \psi$-regular if and only if its $\psi-T_{0}$-identification space $\left(X_{\psi}, Q\left(X_{\psi}\right)\right)$ is $\varphi \psi$-regular, where $\varphi \leq \psi$.

Proof. $(\Rightarrow)$ Let $A$ be $\varphi$-closed set in $X_{\psi}$ and let $C_{x} \notin A$, where $C_{x}$ is the equivalence class containing $x$. Since $P_{\psi}$ is continuous and open, then $P_{\psi}^{-1}(A)$ is $\varphi$-closed set in $X$ not containing $x$. Then there exist disjoint $\psi$-open sets $U$ and $V$ such that $x \in U$ and $P_{\psi}^{-1}(A) \subseteq V$. Since $P_{\psi}$ is continuous, open and $P_{\psi}^{-1}\left(P_{\psi}(U)\right)=U$ for all $U \in \psi O(X)$, then $P_{\psi}(U)$ and $P_{\psi}(V)$ are disjoint $\psi$-open sets in $X_{\psi}$ such that $P_{\psi}(x)=C_{x} \in P_{\psi}(U)$ and $A \subseteq P_{\psi}(V)$.

$(\Leftarrow)$ Let $A$ be disjoint $\varphi$-closed set in $X$ not containing $x$. Since $P_{\psi}$ is continuous, open and $P_{\psi}^{-1}\left(P_{\psi}(A)\right)=A$ for all $A \in \psi O(X)$, then $P_{\psi}(A)$ is $\varphi$-closed set in $X_{\psi}$ and $C_{X} \notin P_{\psi}(A)$. Then there exist disjoint $\psi$-open sets $U$ and $V$ in $X_{\psi}$ such that $C_{X} \in U$ and $P_{\psi}(A) \subseteq V$ and $P_{\psi}^{-1}(U)$ and $P_{\psi}^{-1}(V)$ are disjoint $\psi$-open sets in $X$ containing $x$ and $A$, respectively.

Using Table 2.1, Theorem 3.7 unifies several known results. For example, if $\varphi=\psi=$ cl $\circ$ int, then it yields Theorem 2.1 ((a) $\Leftrightarrow$ (b)) of Dorsett [14]. Similarly, with $\varphi=$ int, $\psi=$ cl $\circ$ int it yields Theorem 2.1 of Dorsett [15].

Theorem 3.8. A space $(X, \tau)$ is $\psi$-normal, then $(X, \tau)$ is $\varphi \psi$-normal, where $\varphi \leq \psi$.

Proof. Let $A$ and $B$ be disjoint $\varphi$-closed sets in $X$. Since $\varphi \leq \psi, A$ and $B$ are disjoint $\psi$-closed sets and then there exist disjoint $\psi$-open sets $U$ and $V$ such that $A \subseteq U$ and $B \subseteq V$.

Referring to Table 2.1, Theorem 3.8 contains several results in the literature. For example, with $\varphi=$ int, $\psi=\mathrm{cl} \circ$ int it gives that if a space $(X, \tau)$ is semi-normal, then $(X, \tau)$ is $s$-normal, a result due to Dorsett [17].

Example 2.1 [17] shows that converse of the Theorem 3.8 is false.

THEOREM 3.9. A space $(X, \tau)$ is $\psi$-regular, then $(X, \tau)$ is $\varphi \psi$-regular, where $\varphi \leq \psi$.

Proof. The proof is similar to that of Theorem 3.8.

Referring to Table 2.1, Theorem 3.9 contains several results in the literature. For example, with $\varphi=$ int, $\psi=\mathrm{cl} \circ$ int it gives that if a space $(X, \tau)$ is semi-regular, then $(X, \tau)$ is $s$-regular, a result due to Dorsett [14].

Example 3.2 [11] shows that converse of the Theorem 3.9 is false.

LEMMA 3.10. If $(X, \tau)$ is semicompact $R_{0}$, then $(X, \tau)$ is E.D.

Proof. Let $A$ be an open set in $X$. Since every open set is also closed [17, Theorem 2.4], $A \subseteq \operatorname{int}(\operatorname{cl} A)$ and $\operatorname{cl} A \subseteq \operatorname{int}(\operatorname{cl} A)$. Therefore, we obtain $\operatorname{cl} A$ is open set in $X$.

In [4], it was shown that for a subset $A$ of $(X, \tau)$, int $A \subseteq \alpha \operatorname{int} A \subseteq p \operatorname{int} A \subseteq \beta \operatorname{int} A$, $\operatorname{int} A \subseteq \alpha \operatorname{int} A \subseteq \operatorname{sint} A \subseteq \beta \operatorname{int} A$, and in [16], int $A \subseteq f \operatorname{int} A \subseteq s \operatorname{int} A \subseteq \beta \operatorname{int} A$, where $\alpha \operatorname{int} A$ (respectively, $f \operatorname{int} A, s \operatorname{int} A, p \operatorname{int} A$, and $\beta \operatorname{int} A$ ) is $\alpha$-interior (respectively, feebly-interior, semi-interior, pre-interior, and $\beta$-interior) of $A$. Later in [3], it was shown that if $(X, \tau)$ is submaximal and E.D., then $\tau=\beta O(X)=\{A \subseteq X \mid A$ is $\beta$-open set $\}$. Therefore, by Lemma 3.10, if $(X, \tau)$ is semicompact $R_{0}$ and submaximal, $\tau=\beta O(X)=$ $\{A \subseteq X \mid A$ is $\beta$-open set $\}$. Consequently, we obtain the following. 
COROLlary 3.11. If $(X, \tau)$ is semicompact $R_{0}$ and submaximal, then all of the $\varphi \psi$ normality and $\varphi \psi$-regularity for the $(X, \tau)$ are equivalent.

The next result follows immediately from Theorem 2.4 [17] and Corollary 3.11.

COROLlary 3.12. If $(X, \tau)$ is semicompact $R_{0}$ and submaximal, then the following are equivalent:

(1) $(X, \tau)$ is $\varphi \psi$-normal,

(2) $(X, \tau)$ is $\varphi \psi$-regular,

(3) $\{\varphi \operatorname{cl}\{x\} \mid x \in X\}$ is finite,

(4) $(X, \tau)$ is $s$-weakly Hausdorff,

(5) $(X, \tau)$ is semi- $R_{1}$,

(6) $(X, \tau)$ is $R_{1}$,

(7) $(X, \tau)$ is completely regular.

ACKNOWLEDGEMENT. This research was supported by the Research Foundation of Cumhuriyet University.

\section{REFERENCES}

[1] M. E. Abd El-Monsef, S. N. El-Deeb, and R. A. Mahmoud, $\beta$-open sets and $\beta$-continuous mapping, Bull. Fac. Sci. Assiut Univ. A 12 (1983), no. 1, 77-90. MR 87b:54002. Zbl 577.54008.

[2] M. E. Abd El-Monsef, A. N. Geaisa, and R. A. Mahmoud, $\beta$-regular spaces, Proc. Math. Phys. Soc. Egypt (1985), no. 60, 47-52 (1987). MR 89f:54042. Zbl 663.54015.

[3] M. E. Abd El-Monsef and M. A. Kozea, On extremally diconnectedness, ro-equivalence and properties of some maximal topologies, 4th Conf. Oper. Res. and Math. Method (Alex. Univ.), 1988.

[4] M. E. Abd El-Monsef, R. A. Mahmoud, and E. R. Lashin, $\beta$-closure and $\beta$-interior, J. Fac. Educ., Sec. A, Ain Shams Univ. 10 (1986), 235-245. Zbl 616.54001.

[5] N. Bourbaki, Elements of Mathematics. General Topology. Part 1, Hermann, Paris; Addison-Wesley Publishing Co., Reading, Mass. London, Don Mills, Ont., 1966. MR 34\#5044a. Zbl 145.19302.

[6] _ Elements of Mathematics. General Topology. Part 2, Hermann, Paris; Addison-Wesley Publishing Co., Reading, Mass. London, Don Mills, Ont., 1966. MR 34\#5044b. Zbl 145.19302.

[7] Á. Császár, Generalized open sets, Acta Math. Hungar. 75 (1997), no. 1-2, 65-87. MR 98e:54001. Zbl 924.54003.

[8] A. S. Davis, Indexed systems of neighborhoods for general topological spaces, Amer. Math. Monthly 68 (1961), 886-893. MR 35\#4869. Zbl 106.15504.

[9] C. Dorsett, Semi- $T_{2}$, semi- $R_{1}$, and semi- $R_{0}$ topological spaces, Ann. Soc. Sci. Bruxelles Sér. I 92 (1978), no. 3, 143-150. MR 80a:54026. Zbl 408.54009.

[10] _,$T_{0}$-identification spaces and $R_{1}$ spaces, Kyungpook Math. J. 18 (1978), no. 2, 167174. MR 80a:54027. Zbl 408.54008.

[11] _ s-weakly Hausdorff spaces, Bull. Soc. Math. Grèce (N.S.) 21 (1980), 3-11. MR 86g:54033. Zbl 522.54018.

[12] _ Semicompact $R_{1}$ and product spaces, Bull. Malaysian Math. Soc. (2) 3 (1980), no. 1, 15-19. MR 82a:54014. Zbl 452.54014.

[13] _ Semi-T $T_{0}$-identification spaces, semi-induced relations, and semiseparation axioms, Bull. Calcutta Math. Soc. 73 (1981), no. 2, 81-86. MR 83i:54020. Zbl 492.54007.

[14] _ Semiregular spaces, Soochow J. Math. 8 (1982), 45-53. MR 84d:54040. Zbl 527.54017.

[15] _ s-regular and s-normal spaces, Math. Nachr. 115 (1984), 265-270. MR 85m:54015. Zbl 562.54006 . 
[16] _ Feebly separation axioms, the feebly induced topology, and separation axioms and the feebly induced topology, J. Karadeniz Univ. Fac. Arts Sci. Ser. Math. Phys. 8 (1985), 43-54. MR 89g:54048. Zbl 601.54021.

[17]__ Seminormal spaces, Kyungpook Math. J. 25 (1985), no. 2, 173-180. MR 87d:54038. Zbl 593.54019.

[18] _ s-separable spaces, feebly continuous functions, and feebly separation axioms, Ganit 11 (1991), no. 1-2, 19-24. MR 95a:54036. Zbl 818.54022.

[19] N. El-Deeb, I. A. Hasanein, A. S. Mashhour, and T. Noiri, On p-regular spaces, Bull. Math. Soc. Sci. Math. R. S. Roumanie (N.S.) 27(75) (1983), no. 4, 311-315. MR 85d:54018. Zbl 524.54016.

[20] N. Levine, Semi-open sets and semi-continuity in topological spaces, Amer. Math. Monthly 70 (1963), 36-41. MR 29\#4025. Zbl 113.16304.

[21] S. N. Maheshwari and R. Prasad, On s-regular spaces, Glasnik Mat. Ser. III 10(30) (1975), no. 2, 347-350. MR 53\#1518. Zbl 316.54017.

[22] _ Some new separation axioms, Ann. Soc. Sci. Bruxelles Sér. I 89 (1975), no. 3, 395402. MR 52\#6660. Zbl 302.54023.

[23] _ On s-normal spaces, Bull. Math. Soc. Sci. Math. R. S. Roumanie (N.S.) 22(70) (1978), no. 1, 27-29. MR 58\#2714. Zbl 373.54016.

[24] S. N. Maheshwari and U. Tapi, Note on some applications on feebly open sets, Madhya Bharati J. Un. Saugar (1978-1979).

[25] R. A. Mahmoud and M. E. Abd El-Monsef, $\beta$-irresolute and $\beta$-topological invariant, Proc. Pakistan Acad. Sci. 27 (1990), no. 3, 285-296. MR 92b:54001.

[26] A. S. Mashhour, I. A. Hasanein, and S. N. El-Deeb, A note on semicontinuity and precontinuity, Indian J. Pure Appl. Math. 13 (1982), no. 10, 1119-1123. MR 84a:54021. Zbl 499.54009.

[27] T. M. Nour, Contrubutions to theory of bitopological spaces, Ph.D. thesis, Univ. of Delhi, 1989.

[28] S. Willard, General Topology, Addison-Wesley Publishing Co., Reading, Mass., London, Don Mills, Ont., 1970. MR 41\#9173. Zbl 205.26601.

MAHIDE KÜÇÜK: DEPARTMENT OF MATHEMATICS, FACULTY OF SCIENCE, ANADOLU UNIVERSITY, 26470 ESKISSEHIR, TURKEY

E-mail address: mkucuk@anadolu.edu.tr

İdris Zorlutuna: Department of MATHematics, Faculty of SCIENCE, CUMHURIYeT UNIVERSITY, 58140 SİVAS, TURKEY

E-mail address: izorlutuna@hotmai 1.com 


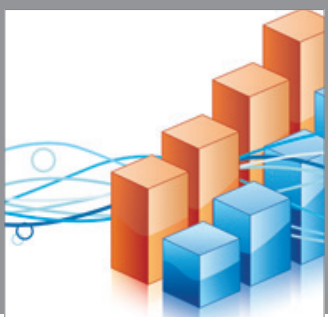

Advances in

Operations Research

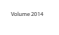

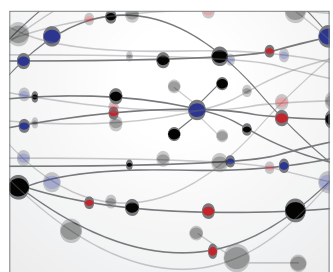

\section{The Scientific} World Journal
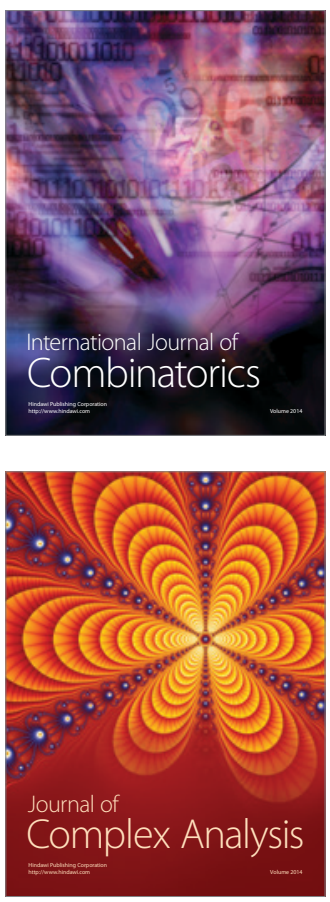

International Journal of

Mathematics and

Mathematical

Sciences
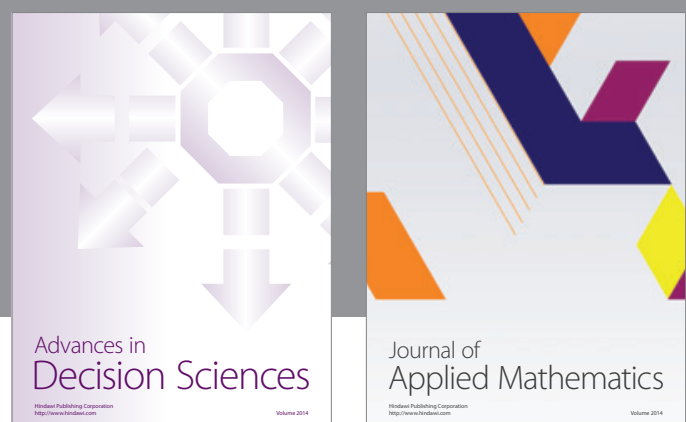

Journal of

Applied Mathematics
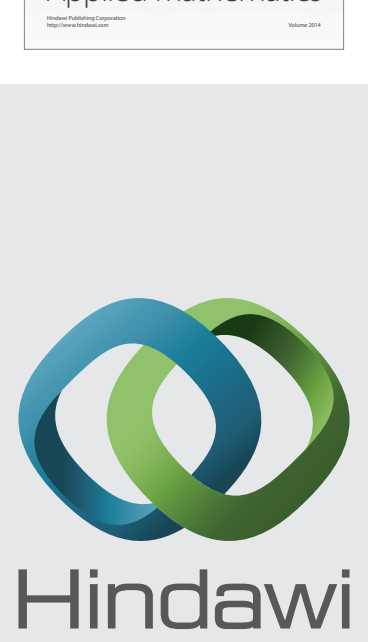

Submit your manuscripts at http://www.hindawi.com
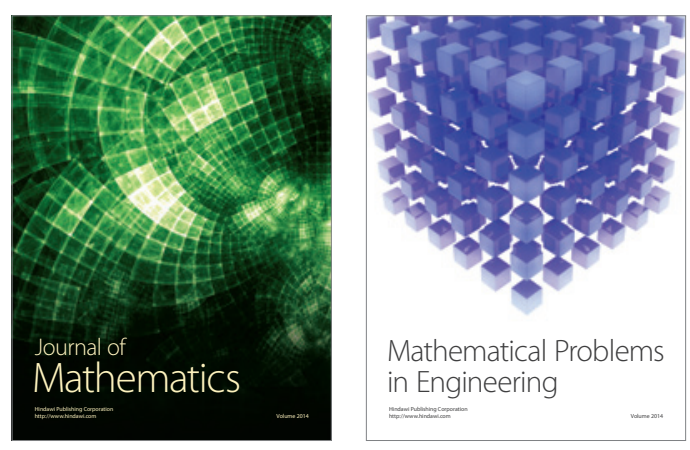

Mathematical Problems in Engineering
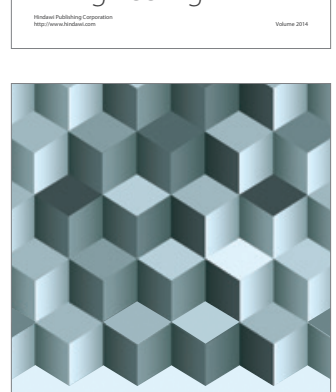

Journal of

Function Spaces
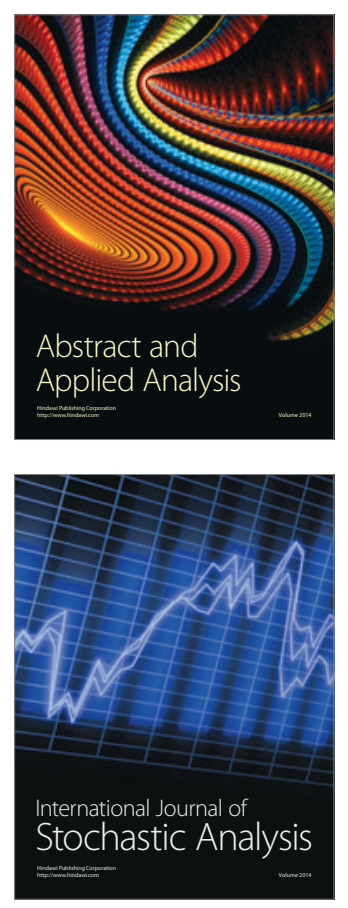

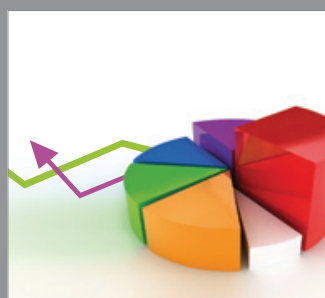

ournal of

Probability and Statistics

Promensencen
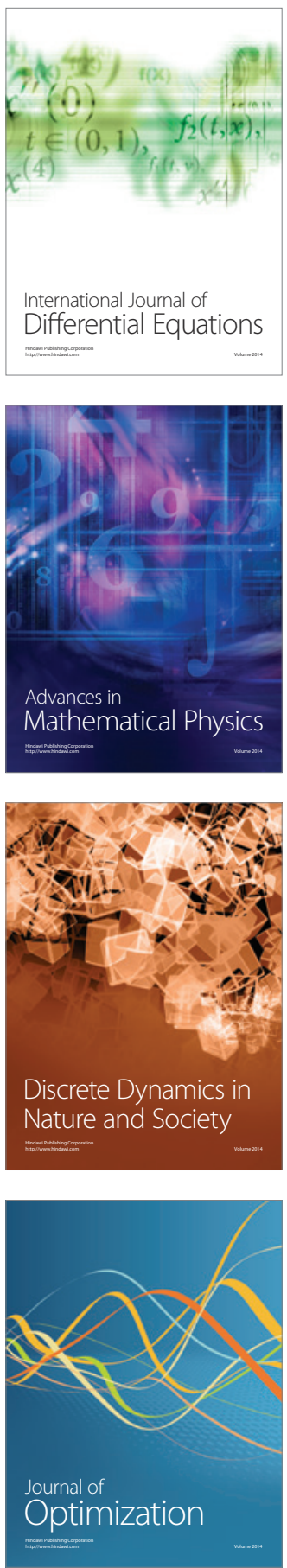